\title{
Voice Operated Tool- Examination Portal for Blind Persons
}

\author{
Akriti Vats \\ Students of BTech(IT) final \\ year, \\ Department of Information \\ Technology
}

\author{
Apoorv Tandon \\ Students of BTech(IT) final \\ year, \\ Department of Information \\ Technology
}

\author{
Deepam Varshney \\ Students of BTech(IT) final \\ year, \\ Department of Information \\ Technology
}

\author{
Amit Sinha \\ Associate Professor \\ Department of Information Technology \\ ABES Engineering College, Ghaziabad
}

\begin{abstract}
Speech recognition applications are becoming more and more useful now a days. Various interactive speech aware applications are available in the market. But they are usually meant for and executed on the traditional general purpose computers. With growth in the needs for embedded computing and the demand for emerging embedded platforms, it is required that the Speech Recognition System (SRS) are available on them too. In this paper, we are presenting a desktop application named as Examination Portal for Blind Persons so as to eliminate the use of an assistant while giving a multiple choice question type examination. Further this tool can be implemented as a mobile application and a web based application.
\end{abstract}

\section{Keywords}

Speech recognition

\section{INTRODUCTION}

Speech Recognition (SR) is the translation of spoken words into text. It is also known as "Automatic Speech Recognition" (ASR), "Computer Speech Recognition" (CSR) or just "Speech to Text" (STT). Speech Recognition is not providing ease in accessing such systems to only blind people but also who have partial vision and differently able people.

In last few years due to continuous incremental improvements, speech recognition has become a strong medium to translate spoken words into texts. Various softwares like Google Voice and Siri have allowed mobile users to just use their voice to operate their cell phones.

The several research papers have been published so far have no real work and no such successful implementation in this field. This paper is an initiative to design a voice operated tool which aims to make differently able person independent, so that they can perform in their examination by themselves, without the help of any assistant. This tool can be implemented as a mobile or web application in future.

The accuracy of the tool depends upon the hardware we are using along with the environment as different microphones have different frequencies and hertz of input. Along with that the system only recognizes UK language and a peaceful environment with no source of extra noises.

\section{OBJECTIVE}

1. The basic objective is to design a framework for the upliftment of blind users.

2. To design a database for handling various modules like Question Bank Candidate details, result analysis.

3. To develop an automated tool through which the intelligence level of blind persons may be tested.

\section{METHODOLOGY}

A Voice Operated Tool (VOT) is a device controlled by the human voice. These types of tools are used for different applications. Here, we are focussing on a device needed for blind people.

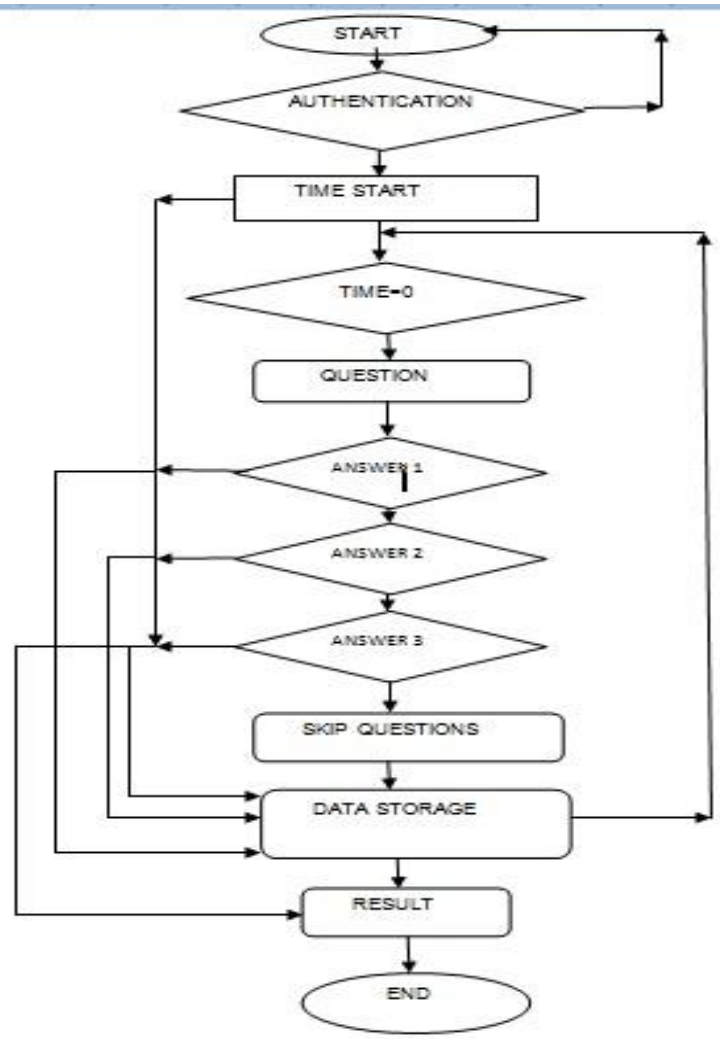

Fig 1: The steps for the development Voice tool 
The Aptitude test will be taken on this tool in following steps:-

1. The user is provided with the hardware equipment i.e. Headphone and microphone, and with the system.

2. Authenticate User's Login by validating the Thumb Impression--Save some Thumb impressions already in the system or the software and use these impressions for validation without using Biometric Device.

3. Create an administrator who will manage all the information of the user. He can create user's account, delete and view the user's test results.

4. Software Working should be like

- The user speaks out his name and then he is validated by his thumb impression.

- System will welcome the user by saying Welcome xyz.

- User will give the instruction for starting the test and the test should get started.

- Text to speech software working at the Backend will take a random question from the SQL Database and speak out the question with the options to the user.

- The user will then speak out one option which will be activated by the speech to text software working at the backend.

- When user says Next system should move to the next question and when he says Finish the system should stop the test. A repeat option should also be added so that if the user doesn't listen properly to the question first time, he or she has an option to repeat it.

- A timer for the whole test should be set for specific time. After that time the test should end automatically.

- The Result of the test is then generated and saved in the user's database and also the result is spoken out to the user.

\section{IMPLEMENTATION}

The Implementation can be done in five modules:

1. LOGIN PAGE- The administrator can login through voice as well us through typing but the differently able candidate has to login through voice. There is an option to reset the password in case system has not taken the correct input. The narrator works as medium between tool and user.

2. QUESTION WINDOW- After the candidate is authenticated, a set of questions appear on the screen. The questions come randomly from the large database of questions along with their four options including the correct one which are all previously set by the admin.

3. EXAM SCHEDULE and TIMER- There is a set timer or a time clock which is being set previously by the admin and the exam initiation starts and ends as per the schedule.

4. RESULT- The window immediately displays and narrator speaks out the result reflecting the performance of the candidate as soon as the test is over.

5. DATABASE- The overall objective in the development of database has been to treat data as an organizational resource.

The design of system-

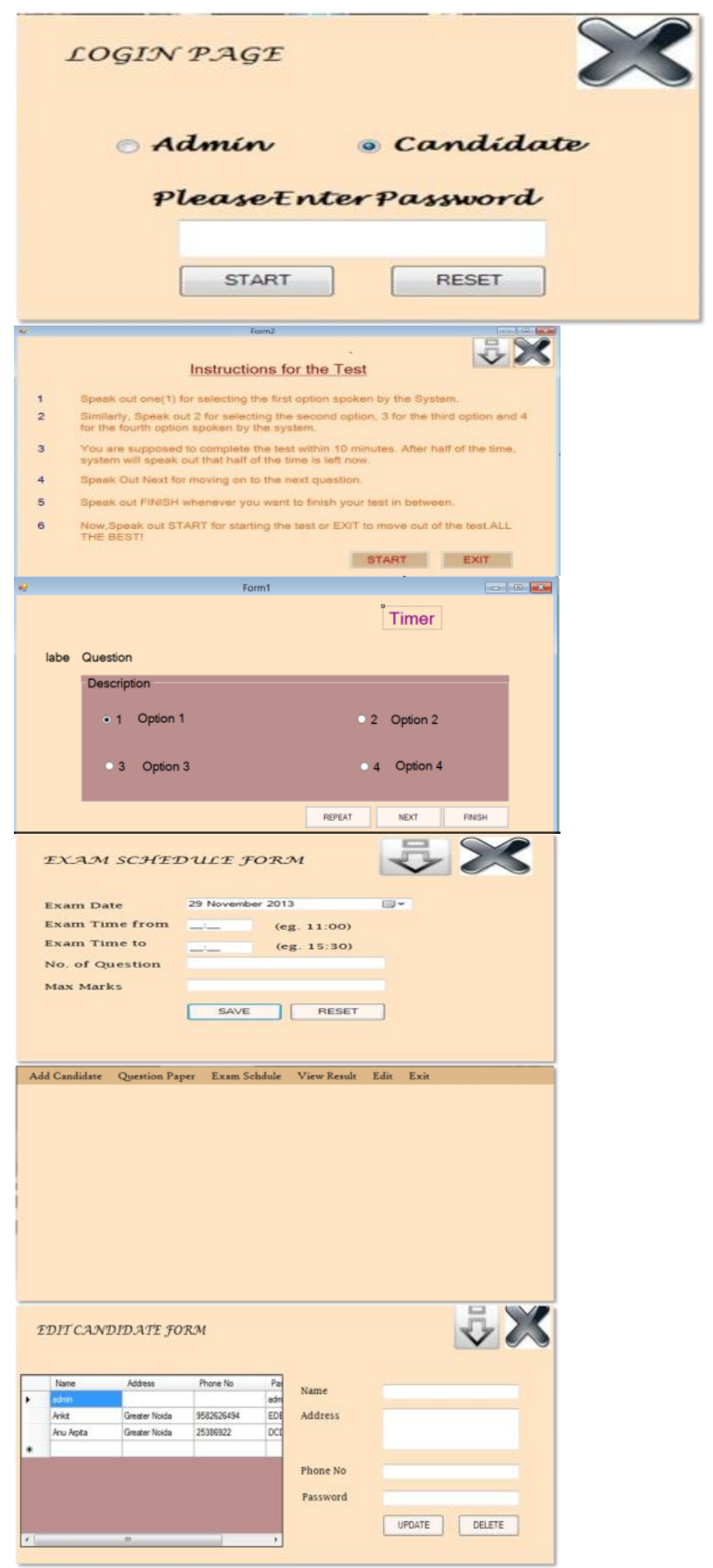

Fig. 2: Snap shots of developed tool

\section{CONCLUSION}

Nowadays, Speech recognition is used extensively for many purposes, but speech recognition is especially very useful for people who have difficulty in using their hands, ranging from mild repetitive stress injuries to involved disabilities that preclude using conventional computer input devices. Speech recognition provides best way for such people to give exams. Through speech recognition, instead of pressing buttons on keyboards one can easily operate a computer system by just using his/her voice. This tool provides a way for the people who 
cannot use their hands or cannot see to give exams by just using their voices.

This tool works on voice recognition and provides a way for user to give examination. It aims to recognise the user through voice input and conduct aptitude exams type-free with the help of microphone and head phones only. The research for more accurate results is still on, in the field of voice operated tools. The future for the recognition of voice and speech is wide spread as more and more applications are now being made using speech recognition tool.

\section{REFERENCES}

[1] B. Shanmuga Sundari, Essaki Durai.K, Srinivasan,.PET Engineering College, Vallioor, Journal and volume:"international journal of technology enhancements and emerging engineering research, vol 2, issue 569 issn $2347-4289 "$
[2] Susumu Harada , Jacob O. Wobbrock James A. Landay (University Of Washington) Voice Draw: A Hands- Free Voice Driven Application For People With Motor Impairments , 2007 ACM New York, USA

[3] M.A.Anusuya and S.K.Katti (Sri Jayachamarajendra College OF Engineering, Mysore), Speech Recognition By a Machine: A Review , : International Journal Of Computer Science and Information Security (2009)

[4] National Braille Association Tape Recording Manual, National Library Service for the Blind \& Physically Handicapped, Library of Congress, Washington, DC.

[5] "Bridging the Gap: Best Practices for Instructing Adults Who Are Visually Impaired and Have Low Literacy Skills" American Foundation for the Blind, 2002 